\title{
A RELATION CONNECTING THE DERIVATIVES OF PHYSICAL QUANTITIES ${ }^{1}$
}

\author{
By Mayo D. Hersey
}

CONTENTS

I. Scope of the paper...............................

2. Statement of the problem ............................ 22

3. Other classes of relations among derivatives................. 22.

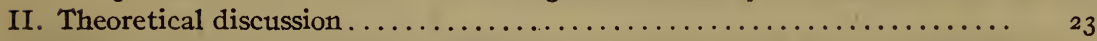

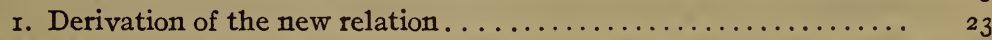

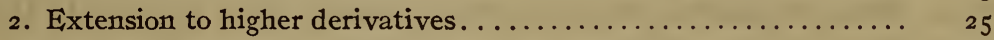

3. Integral form of the relation ........................... 25

4. Discussion of the constant-product restriction . . . . . . . . . 26

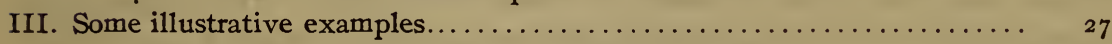

r. Variation of journal friction with size of bearing ............. 2 \%

2. Effect of gravity on a rolling-ball viscosimeter............. 28

3. Effect of high pressure on accuracy of a rolling-ball viscosimeter.. 29

\section{INTRODUCTION}

I. Scope of the Paper.-In this paper it is shown how the theory of dimensions may be used in a differential form; a procedure which appears fruitful particularly in investigating the effect of given sources of error on the performance of measuring instruments.

The examples which led to the necessity for developing this method are discussed at the end of the paper and illustrated by experimental data.

2. Statement of the Problem.-Given the fact that some relation of unknown form

$$
Q_{0}=f\left(Q_{1}, Q_{2}, \quad \cdot \quad \cdot \quad Q_{\mathrm{N}-1}\right)
$$

subsists between $N$ physical quantities $Q_{0}, Q_{1}, Q_{2}, \quad$. . . $Q_{N-1}$, no others being involved, it is required to deduce a relation of known form

$$
\frac{\partial Q_{0}}{\partial Q_{1}}=F\left(\frac{\partial Q_{0}}{\partial Q_{2}}, Q_{0}, Q_{1}, Q_{2}, \quad . \quad . \quad \cdot Q_{\mathrm{N}-1}\right)
$$

\footnotetext{
1 This work was done at the Jefferson Physical Laboratory, Harvard University, in I9I6, and presented as an interesting application of Buckingham's II theorem during a series of four lectures on Dimensional Reasoning given at the Friday evening conferences. It was first published in the Journal Wash. Acad. Sci., 6, pp. 620-629, 1916, and is reprinted here to provide the mathematical basis for a forthcoming paper on the effect of temperature on bodies of constant shape.
}

$$
111675^{\circ}-19
$$


such that at any point whose generalized coordinates, $Q_{0}, Q_{1}, Q_{2}$, etc., are given, the value of any one of the $N-I$ partial derivatives of $Q_{0}$ can be computed from some other one. Thus, it is required to calculate one of the component slopes of the generalized surface (I) from a knowledge of another, although the equation of the surface is not available. The interest of the problem to the physicist lies in the fact that he may wish to learn the value of a derivative not readily accessible to experiment, in a case where some other derivative of the same quantity can easily be observed. It will be shown that a definite solution can always be obtained, provided certain dimensionless products of the $N$ quantities are held constant.

3. Other Claisses of Relations Among Derivatives.-The proposition, that relations may be found connecting the derivatives of quantities in the absence of a primitive equation, is not new. There are two other classes of such relations. One consists of mathematical identities, applicable to any set of related quantities, whether physical or not. To this class belongs the identity

$$
\frac{\partial}{\partial Q_{1}} \frac{\partial Q_{0}}{\partial Q_{2}}=\frac{\partial}{\partial Q_{2}} \frac{\partial Q_{0}}{\partial Q_{1}}
$$

as well as the triple product relation

$$
\frac{\partial Q_{0}}{\partial Q_{1}} \cdot \frac{\partial Q_{1}}{\partial Q_{2}} \cdot \frac{\partial Q_{2}}{\partial Q_{0}}=-1
$$

The other class comprises relations requiring the explicit use of physical laws, such as the two laws of thermodynamics, or Hamilton's principle. To this class belong Maxwell's four thermodynamic relations, and the reciprocal relations of generalized dynamics. $^{2}$ The relations to be presented here are of a nature intermediate between the other two classes, in that they require a knowledge only of the dimensions of the quantities.

\section{THEORETICAL DISCUSSION.}

I. Derivation of the New Relation.-The present result depends upon and is a corollary to Buckingham's $\Pi$-theorem, ${ }^{3}$ according to which any complete physical equation is reducible to the form

$$
\text { funct. }\left(\Pi_{1}, \Pi_{2}, \quad \text {. . . } \Pi_{i}\right)=0
$$

\footnotetext{
$8 \mathrm{~J}$. J. Thomson, Applications of dynamics to physics and chemistry, Chap. 5.

${ }^{3}$ Journal Wash. Acad. Sci., 4, pp. 347-353, 1914; Phys. Rev., 4, pp. 345-376, 1914; Trans. Am. Soc. Mech. Engs., 37. pp. 263-296; 1915. Anyone who can sufficiently visualize the meaning of the II-theorem will be able to treat each concrete problem by itself, dispensing with the formulas of the present paper save as a check.
} 
in which the $\Pi$ 's are all the independent dimensionless products which can be built up by combining in any way the $N$ physical quantities involved. Further, the total number of such products, or dimensionless arguments, will always be the same, no matter how the quantities are grouped. This number will be

$$
i=N-k
$$

if $k$ is the number of fundamental units needed for measuring the $N$ quantities. ${ }^{4}$

Let $\Pi_{0}$ and $\Pi$ designate any two of the $i$ products in (5) which contain between them the three quantities $Q_{0}, Q_{1}$, and $Q_{2}$ in which we are interested. Let $Q_{0}$ appear to the first power in $\Pi_{0}$ and not at all in any other product, which can always be done, for Buckingham has shown that a certain standard arrangement is possible in which each product contains to the first power some one quantity of type $P$ which occurs nowhere else. ${ }^{5}$ We shall then have

and

$$
\Pi_{0}=Q_{1}^{a_{0}} Q_{2}^{\beta_{0}} \ldots Q_{\mathbf{k}}^{x_{0}} \cdot Q_{0}
$$

$$
\Pi=Q_{1}^{a} Q_{2}^{\beta} \ldots Q_{\mathrm{k}}^{\mathrm{k}} \cdot Q_{\mathrm{k}+1}
$$

The exponents are abstract numbers fixed by the dimensions of the $N$ quantities; in any particular problem some of them may be zero. If we now agree to keep the remaining $i-2$ products constant, (5) becomes

$$
\Pi_{0}=\phi(\Pi)
$$

in which the form of $\phi$ is unknown. The restriction to constant products can always be fulfilled in theory, but it may lead to difficulties in practice; it will be discussed in a later section. Differentiating (9) and then (8) gives in succession

From (7)

$$
\frac{\partial \Pi_{0}}{\partial Q_{1}}=\frac{d \phi}{d \Pi} \cdot \frac{\partial \Pi}{\partial Q_{1}}=\frac{d \phi}{d \Pi} \frac{a \Pi}{Q_{1}}
$$

$$
\frac{\partial \Pi_{0}}{\partial Q_{1}}=\frac{\partial Q_{0}}{\partial Q_{1}} \frac{\Pi_{0}}{Q_{0}}+\frac{a_{0} \Pi_{0}}{Q_{1}}
$$

Comparing (IO) and (II)

$$
\Pi \frac{d \phi}{d \Pi}=\frac{Q_{1} \Pi_{0}}{a}\left(\frac{\mathrm{I}}{Q_{0}} \frac{\partial Q_{0}}{\partial Q_{1}}+\frac{a_{0}}{Q_{1}}\right)
$$

4 The question of the number of fundamental units needed has been discussed by Riabouchinsky, Rayleigh, and Buckingham; see Nature, 96, pp. 396-397; 1915.

3 Trans. Am. Soc. Mech. Engs., 37, pp. 291-292; note equation (II) and its discussion. 
Similarly

$$
\Pi \frac{d \phi}{d \Pi}=\frac{Q_{2} \Pi_{0}}{\beta}\left(\frac{\mathrm{I}}{Q_{0}} \frac{\partial Q_{0}}{\partial Q_{2}}+\frac{\beta_{0}}{Q_{2}}\right)
$$

Comparing (12) and (13)

$$
\frac{\partial Q_{0}}{\partial Q_{1}}=\left(\frac{a}{\beta} \beta_{0}-a_{0}\right) \frac{Q_{0}}{Q_{1}}+\frac{a}{\beta} \frac{Q_{2}}{Q_{1}} \frac{\partial Q_{0}}{\partial Q_{2}}
$$

Hence the desired relation (2) has the linear form

$$
\frac{\partial Q_{0}}{\partial Q_{1}}=a+b \frac{\partial Q_{0}}{\partial Q_{2}}
$$

in which the coefficients

and

$$
a=\left(\frac{a}{\beta} \beta_{0}-a_{0}\right) \frac{Q_{0}}{Q_{1}}
$$

$$
b=\frac{a}{\beta} \frac{Q_{2}}{Q_{1}}
$$

involve none of the $N$ quantities save $Q_{0}, Q_{1}$, and $Q_{2}$.

Evidently ( 14 ) can be written also

$$
\frac{\partial \log Q_{0}}{\partial \log Q_{1}}=\left(\frac{a}{\beta} \beta_{0}-a_{0}\right)+\frac{a}{\beta} \frac{\partial \log Q_{0}}{\partial \log Q_{2}}
$$

in which the coefficients are independent of the coordinates. Thus the relation connecting the logarithmic derivatives is the same all over the generalized surface.

If no two independent products can be found which do contain between them the three quantities $Q_{0}, Q_{1}$, and $Q_{2}$, either $a$ or $\beta$ will vanish, showing that the derivatives are independent. Thus, while it is always possible to find a relation connecting any derivative with some other, it is not always possible to find a relation connecting a given derivative with any other desired.

2. Extension to Higher Derivatives.-Differentiating (14) with respect to $Q_{1}$ and using the identity (3) gives

$$
\frac{\partial^{2} Q_{0}}{\partial Q_{1}{ }^{2}}=A+B \frac{\partial Q_{0}}{\partial Q_{2}}+C \frac{\partial^{2} Q_{0}}{\partial Q_{2}{ }^{2}}
$$

in which the coefficients are

and

$$
\begin{gathered}
A=\frac{Q_{0}}{Q_{1}{ }^{2}}\left(\frac{a}{\beta} \beta_{0}-a_{0}\right)\left(\frac{a}{\beta} \beta_{0}-a_{0}-\mathrm{I}\right) \\
B=\frac{Q_{2}}{Q_{1}{ }^{2}} \frac{a}{\beta}\left(\frac{a}{\beta}-\mathrm{I}\right)\left(\mathrm{I}+2 a_{0}\right)
\end{gathered}
$$

$$
C=\left(\frac{Q_{2} a}{Q_{1} \beta}\right)^{2}
$$


Thus the curvature with respect to $Q_{1}$ can be calculated from the slope and the curvature with respect to $Q_{2}$.

3. Integral Form of the Relation. ${ }^{6}$-Integrating (I4) at the point $\left(Q_{0}=q_{0}, Q_{1}=q_{1}, Q_{2}=q_{2}\right)$ over an interval so short that $\frac{\partial Q_{0}}{\partial Q_{2}}$ may be treated as constant, and denoting its value by the symbol $\frac{\partial q_{0}}{\partial q_{2}}$, gives for the primitive equation of an element of the surface

in which

$$
\frac{Q_{0}}{q_{0}}=\left(\frac{Q_{1}}{q_{1}}\right)^{\mathrm{h}}
$$

$$
h=\frac{a}{\beta}\left(\frac{q_{2}}{q_{0}} \frac{\partial q_{0}}{\partial q_{2}}+\beta_{0}\right)-a_{0}
$$

The use of (23).would permit a direct comparison of any new results obtained by the present method with empirical results previously published in one-term, constant-exponent formulas.

4. Discussion of the Constant-Product Restriction.-Let $\Pi_{c}$ denote any one of the $i-2$ arguments which we have agreed to hold constant, and let $Q$ stand for either $Q_{1}$ or $Q_{2}$. Then, unless $\Pi_{\mathrm{c}}$ can be so chosen that it does not contain $Q$, it must be so chosen that it will contain some additional quantity $Q_{\mathrm{c}}$ not occurring in any other product. The rule for keeping $\Pi_{c}$ constant will then be: Vary $Q_{0}$ simultaneously in such a manner as to compensate the changes due to $Q$.

If $Q$ enters $\Pi_{\mathrm{c}}$ to the $n^{\text {th }}$ power and $Q_{\mathrm{c}}$ enters it to the first, the derivatives in (I5) and elsewhere are subject to one or more conditions of the type $Q_{\mathrm{c}} \propto Q^{-\mathrm{n}}$. For such a derivative let us adopt from now on the notation $\left(\frac{\partial Q_{0}}{\partial Q}\right)_{Q_{c} \propto Q^{-a}}$. There are two experimentally independent methods for getting its numerical value: First, by directly observing the change in $Q_{0}$ with $Q$ while simultaneously changing $Q_{\mathrm{c}}$ in the prescribed manner; second, by calculating it from separate observations on the change in $Q_{0}$ with $Q$ at constant $Q_{\mathrm{c}}$, and the change in $Q_{0}$ with

\footnotetext{
${ }^{6}$ If instead of an isolated value of $\frac{\partial Q_{0}}{\partial \dot{Q}_{2}}$ we were furnished with the entire curve $Q_{0}=f_{2}\left(Q_{2}\right)$, the direct use of the II-theorem would be preferable, and would give the whole curve $Q_{0}=f_{1}\left(Q_{1}\right)$. If successively furnished with additional curves, $Q_{0}=f_{3}\left(Q_{3}\right)$ and so on, we could gradually build up generalized cross sections of the surface ( $\mathrm{I}$ ) until, when $N-k$ independent curves had been given, we should have the whole of it. The problem of developing empirical equations synthetically has not been treated in the available papers. That problem is a general one, of which the problem of the present paper is a special case; this situation is illustrated by the fact that our final result (23) applies only to an infinitesimal piece of the curve $Q_{0}=f_{1}\left(Q_{1}\right)$.
} 
$Q_{\text {o }}$ at constant $Q$. Expanding the conditioned derivative into the form $\left(\frac{\partial Q_{0}}{\partial Q}\right)_{Q_{\mathrm{c}}}+\left(\frac{\partial Q_{0}}{\partial Q_{\mathrm{c}}}\right)_{Q} \frac{d Q_{\mathrm{c}}}{d Q}$ and taking account of the fixed relation between $Q_{\mathrm{c}}$ and $Q$ leads to the working formula

$$
\left(\frac{\partial Q_{0}}{\partial Q}\right)_{Q_{0} \propto Q^{-\mathrm{a}}}=\left(\frac{\partial Q_{0}}{\partial Q}\right)_{Q_{\mathrm{o}}}-n \frac{Q_{\mathrm{c}}}{Q}\left(\frac{\partial Q_{0}}{\partial Q_{\mathrm{c}}}\right)_{Q}
$$

for the second method. In the most general case where there are $i-2$ arguments to be kept constant, the second term on the right of (25) will be replaced by $-\frac{I}{Q}$ times the summation of $i-2$ terms of the type $n Q_{\mathrm{c}}\left(\frac{\partial Q_{0}}{\partial Q_{\mathrm{c}}}\right)_{Q}$.

While the procedure outlined in this section is always possible and sufficient, it is not always necessary or even desirable. For example: if the number of quantities, $N$, does not exceed the number of fundamental units, $k$, by more than 2, there will be no other arguments than $\Pi_{0}$ and $\Pi$; again, if the remaining $i-2$ arguments do not involve $Q$ (i. e., $Q_{1}$ or $Q_{2}$ ), their constancy will not be disturbed at all by the fact that $Q_{1}$ and $Q_{2}$ do vary. Further expedients for simplifying the work will suggest themselves upon examining each particular case by itself.

\section{SOME ILLUSTRATIVE EXAMPLES}

For reference in solving problems it is convenient to rewrite (5) in the form

$Q_{1}^{\alpha_{0}} Q_{2}^{\beta_{0}} \cdots Q_{\mathrm{k}}^{\alpha_{0}} \cdot Q_{0}=$ funct. $\left(Q_{1}^{\alpha} Q_{2}^{\beta} \cdots Q_{\mathrm{k}}^{\kappa} \cdot Q_{\mathrm{k}+\tau}\right.$, and other $\Pi$ 's $)$

The values of $a, \beta$, etc., can now be read off directly by identifying them with the corresponding numerical exponents in the equation, of type (26), afforded by the particular example in hand.

I. Variation of Journal Friction with Size of Bearing.-In the case of a journal bearing, under certain restrictions, we may expect a relation of type (I) to connect the coefficient of friction $f$, with the viscosity of the lubricant $\mu$, the revolutions per unit time $n$, the bearing pressure $p$, the journal diameter $D$, and the volume of oil $V$ forced through the bearing in unit time. Let it be required to calculate the effect of altering the size of the machine from a test in which nothing is varied but the rate of pumping 
oil through the bearing. It may be shown by the $\Pi$-theorem that

$$
f=\text { funct. }\left(\frac{D^{3} n}{V}, \frac{\mu n}{p}, \text { shape }\right)
$$

Let now $f, D$, and $V$ serve, respectively, as $Q_{0}, Q_{1}$, and $Q_{2}$. Comparing (27) with (26), $a_{0}=0, \beta_{0}=0, a=3, \beta=-\mathrm{I}$; hence, by (I6) and (I 7$), a=0$ and $b=-3 \frac{V}{D}$, or

$$
\frac{\partial f}{\partial D}=-3 \frac{V}{D} \frac{\partial f}{\partial V}
$$

Also, by (20) and (22), $A=0, B=\mathrm{I} 2 \frac{V}{D^{2}}$, and $C=9\left(\frac{V}{D}\right)^{2}$; therefore

$$
\frac{\partial^{2} f}{\partial D^{2}}=12 \frac{V}{D^{2}} \frac{\partial f}{\partial V}+9\left(\frac{V}{D}\right)^{2} \frac{\partial^{2} f}{\partial V^{2}}
$$

Equations (28) and (29) enable us to predict the bearing losses of any slightly larger or smaller machine in the same geometrically similar series. This requirement of geometrical similarity is an instance of the constant-product restriction. The products in this case are the length ratios fixing the shape. The first of the two results in this example, namely, equation (28), follows readily enough from equation (27) without calling in the aid of the present theorem at all. For in differentiating $f$ first with respect to $D$ and then with respect to $V$ the same unknown occurs both times and can be eliminated, leaving the desired relation between $\frac{\partial f}{\partial D}$ and $\frac{\partial f}{\partial V}$. The contemplation of this example, which can be solved by inspection because the left-hand member of equation (27) has only one quantity in it, reveals instructively the meaning of the theorem and shows that it is simple when understood.

2. Effect of Gravity on a Rolling-Ball Viscosimeter.-Let it be required to find the effect of gravity on a rolling-ball viscosimeter in terms of the effect produced by changing the size of the instrument. Let $D, l$, and $\theta$ denote, respectively, the diameter and length of the tube and its angle of inclination to the horizontal, $d$ and $\rho_{0}$ the diameter and density of the ball, $\rho$ and $\mu$ the density and viscosity of the liquid, and $t$ the roll-time ${ }^{7}$ in a locality ${ }^{8}$ of

\footnotetext{
${ }^{7}$ That is, the time required for the ball to roll down. This instrument, proposed by Flowers (Proc. Am. Soc. Test. Mat., 14, pp. $565 ; 1914)$, is further discussed by the writer in Journal Wash. Acad. Sci., 6, pp. 527 ; 1916.

${ }^{8}$ Having set up such a viscosimeter in Cambridge, the question arose whether there would be any sensible change upon taking it to Washington, where gravity is 0.3 per cent less. The conclusion is that the roll-time in a very viscous liquid will be 0.3 per cent greater in Washington; and that the effect of gravity diminishes when the fluidity of the liquid increases, falling to 0.2 per cent for water.
} 
gravity $g$. Assuming that a complete relation does subsist among these quantities, the II-theorem shows that any equation describing that relation, whether obtained theoretically or experimentally, must be reducible to the form

$$
\frac{\mu}{\rho D^{2}} t=\text { funct. }\left(\frac{\rho_{0}}{\rho}, \frac{g \rho^{2} D^{3}}{\mu^{2}}, \text { shape }\right)
$$

the shape, in turn, being fixed by the arguments $\frac{d}{D}, \frac{l}{D}$, and $\theta$. Taking $t, g$, and $D$, respectively, for $Q_{0}, Q_{1}$, and $Q_{2}$ gives $a_{0}=0$, $\beta_{0}=-2, \alpha=\mathrm{I}$, and $\beta=3$; so that by (I 8 )

$$
\frac{g \partial t}{t \partial g}=-\frac{2}{3}+\frac{1}{3} \frac{D}{t} \frac{\partial t}{\partial D}
$$

An interesting check on ( $3 \mathrm{I})$ is afforded by differentiating the empirical equation for such an instrument. ${ }^{9}$ The equation has been presented in the form $y=a+b x$, in which $x$ denotes

$\tau \sqrt{D g\left(\frac{\rho_{0}}{\rho}-\mathrm{I}\right)}$ and $y$ denotes $\nu / \sqrt{D^{3} g\left(\frac{\rho_{0}}{\rho}-\mathrm{I}\right)}, \tau$ being the rolltime per unit length $\frac{t}{l}, \nu$ the kinematic viscosity $\frac{\mu}{\rho}$, and $a$ and $b$ particular numerical values fixed by a particular choice of $\frac{d}{D}$ and $\theta$. Recast in the form (30) it becomes

$$
\frac{\mu}{\rho D^{2}} t=\frac{\mathrm{I}}{b} \cdot \frac{\mathrm{I}}{\frac{\rho_{0}}{\rho}-\mathrm{I}}\left(\mathrm{I}-a \sqrt{\frac{\rho_{0}}{\rho}-\mathrm{I}} \sqrt{\frac{g \rho^{2} D^{3}}{\mu^{2}}}\right) \frac{\mu^{2}}{g \rho^{2} D^{3}} \cdot \frac{l}{D}
$$

or

$$
i=\frac{A}{g D}\left(\mathrm{I}+B \sqrt{g D^{3}}\right)
$$

in which $A$ and $B$ (both intrinsically positive) do not involve $g$ at all, nor $D$ except in a shape factor. The values of $\frac{g}{t} \frac{\partial t}{\partial g}$ and $\frac{D}{t} \frac{\partial t}{\partial D}$ found by differentiating (33) do satisfy (3r).

3. Effect of High Pressure on Accuracy of a Rolling-Ball Viscosimeter.-Without knowing the empirical equation let it be required to predict the change in roll-time due to any small change in liquid density, such as would occur upon using the tube under pressure, by reference to an observation on the effect of changing the ball density. Since an expression for $\frac{\partial t}{\partial \rho}$ in terms of $\frac{\partial t}{\partial \rho_{0}}$ is 
sought, $t, \rho$, and $\rho_{0}$ are selected for $Q_{0}, Q_{1}$, and $Q_{2}$, respectively. If (3o) were to be used as it stands, there would be a restriction on the derivative $\frac{\partial t}{\partial \rho}$, which is hardly to be desired. An equivalent result in a more convenient form can evidently be obtained by confining $\rho$ to a smaller number of arguments. This is done by replacing (30) by one of the alternative forms provided by the II-theorem, such as

$$
\sqrt{\frac{g}{D}} t=\text { funct. }\left(\frac{\rho_{0}}{\rho}, \frac{g \rho_{0}{ }^{2} D^{3}}{\mu^{2}}, \text { shape }\right)
$$

Comparing this with (26), $a_{0}=0, \beta_{0}=0, a=\mathrm{I}, \beta=-\mathrm{I}$; hence by (I4)

or by (25)

$$
\frac{\partial t}{\partial \rho}=-\frac{\rho_{0}}{\rho}\left(\frac{\partial t}{\partial \rho_{0}}\right)_{\mu \propto \rho_{0}}
$$

$$
\frac{\partial t}{\partial \rho}=-\frac{\mathrm{I}}{\rho}\left(\rho_{0} \frac{\partial t}{\partial \rho_{0}}+\mu \frac{\partial t}{\partial \mu}\right)
$$

In the last transformation $\mu$ took the part of $Q_{\mathrm{c}}$ and $\rho_{0}$ of $Q$, while $n$ had the value $-\mathrm{r}$.

The following observations afford an experimental illustration of (36). They were made with a tube $59 \mathrm{~cm}$ long and $\mathrm{I} \mathrm{cm}$ in diameter, containing a one-fourth inch $(0.635 \mathrm{~cm})$ ball, ordinarily of steel $\left(\rho_{0}=7.7 \mathrm{~g} / \mathrm{cm}^{3}\right)$. The tube was filled with lard oil $\left(\mu=0.74\right.$ cgs units, $\left.\rho=0.92 \mathrm{~g} / \mathrm{cm}^{3}\right)$. The slope $\frac{\partial t}{\partial \mu}$ was found to be $3 \mathrm{I}$ cgs units. Substituting now a brass ball $\left(\rho_{0}=8.6 \mathrm{~g} / \mathrm{cm}^{3}\right)$ for the steel one, the roll-time dropped from 27.9 to 24.7 seconds, making $\frac{\partial t}{\partial \rho_{0}}$ equal to $-3.6 \mathrm{cgs}$ units. From these data, in conjunction with $(36)$, the value $\frac{\partial t}{\partial \rho}=5.2 \mathrm{cgs}$ units would be predicted. From (32), the actual value is found to be $5.7 \mathrm{cgs}$ units. Since $\frac{\partial t}{\partial \rho}$ is itself a correction term, the agreement is sufficient.

WASHINGTON, September 23, I9I8. 